\title{
The effectiveness of the sacrospinous hysteropexy for the primary treatment of uterovaginal prolapse
}

\author{
Viviane Dietz • Joyce de Jong • Marieke Huisman • \\ Steven Schraffordt Koops $\cdot$ Peter Heintz • \\ Huub van der Vaart
}

Received: 10 November 2006 / Accepted: 18 February 2007 /Published online: 24 March 2007

(C) International Urogynecology Journal 2007

\begin{abstract}
The objective of this study was to assess the effectiveness of sacrospinous ligament fixation of the uterus as a primary treatment of uterovaginal prolapse. In this observational study, 133 women underwent a sacrospinous hysteropexy. Data were obtained from their medical records, and standardized questionnaires about urogenital symptoms and quality of life were used. All women were invited for gynecological examination, using the Pelvic Organ Prolapse Quantification score. Ninety-nine women responded by returning the questionnaire (mean age, 59.2 and follow-up time, 22.5 months); 60 of these women underwent gynecologic examination. Eighty-four percent of women were highly satisfied about the outcome of the procedure. Serious complications were rare. The recurrence rate of descensus uteri that needed surgical treatment was $2.3 \%$. The recurrence of cystoceles after surgery was $35 \%$, but there were no differences in urogenital symptoms between women with or without a cystocele.
\end{abstract}

Keywords Cystocele - Sacrospinous hysteropexy

Uterovaginal prolapse $\cdot$ Vaginal hysterectomy

\section{Introduction}

In the last decades, many studies showed that sacrospinous ligament fixation is an effective surgical procedure to correct

V. Dietz $(\bowtie) \cdot$ J. de Jong $\cdot$ M. Huisman $\cdot$ P. Heintz $\cdot$ H. van der Vaart Department of Perinatology and Gynecology,

University Medical Center Utrecht,

Utrecht, The Netherlands

e-mail: viviane.dietz@sapio.nl

S. Schraffordt Koops

Department of Gynecology and Obstetrics, Meander Medical Center, Amersfoort, The Netherlands post-hysterectomy vaginal vault prolapse $[1,2]$. Because it has proven its efficacy in vaginal vault prolapse surgery, it might be of interest to use it as a primary technique to correct descensus uteri, the so-called sacrospinous hysteropexy. The anatomical outcome and complication rate of this operation was described in few reports, but most authors do not focus on urogenital symptoms and quality of life after sacrospinous hysteropexy [3-8]. In a previous study by our group, we concluded that the sacrospinous hysteropexy is a promising technique for the correction of descensus uteri [9]. However, the mean follow-up of the study group was relatively short, the postoperative anatomical status was derived from the medical records, and differences in urogenital symptoms in relation to the anatomical outcome were not assessed.

We set out to assess the satisfaction, complications, urogenital symptoms, and quality of life in a group of women after a sacrospinous hysteropexy.

\section{Materials and methods}

Patients

The study group consisted of 133 women who had a vaginal sacrospinous hysteropexy for uterovaginal prolapse in the period January 2000 and June 2004 in three large teaching hospitals in The Netherlands. All women wanted to preserve their uterus. Preoperative cytology of the cervix and ultrasound screening of the uterus and ovaries showed no abnormalities. Data on patient characteristics and perioperative events were collected retrospectively from medical files of all 133 women. All women received a standardized, validated questionnaire in 2005 that covered urogenital symptoms and quality of life aspects. They were invited to 
visit our clinic for a full gynecological examination and Pelvic Organ Prolapse Quantification (POP-Q) assessment. The study was approved by the local ethics committee.

\section{Surgery}

All surgeries were performed by four senior surgeons. The sacrospinous hysteropexy is performed unilaterally to the right ligament. A midline incision in the posterior vaginal wall is extended to the posterior part of the cervix in the midline. Through sharp and blunt dissection, the right sacrospinous ligament is made visible. Two non-absorbable sutures (Prolene 1) are placed through the sacrospinous ligament and subsequently placed through the posterior side of the cervix. An additional classical anterior and/or posterior colporrhaphy (fascia plication), with absorbable vicryl ${ }^{\circledR}$ 2-0 interrupted sutures, was performed when indicated by the judgment of the individual gynecologist. All women were given perioperative antibiotic and thrombosis prophylaxis according to the guidelines from the individual hospitals.

\section{Measurements}

The following data were obtained from the patients' medical files: date of surgery, age at the time of surgery, peri- and postoperative complications, grade of prolapse before surgery, and if additional anterior and/or posterior colporrhaphy were performed. In most cases, the preoperative stage of genital prolapse was still classified according to the halfway system of Baden and Walker, noted as $0=$ no prolapse, $1=$ prolapse halfway to hymen, $2=$ prolapse progressing to hymen, $3=$ prolapse halfway through the hymen, and $4=$ total vaginal prolapse. In the analysis, we dichotomized the grade of prolapse into stage 1 or less and stage 2 or more.

The women who underwent gynecological examination at follow-up were examined by an independent physician trained in POP-Q assessment. The POP-Q score is a reliable and specific method to measure the pelvic organ support [10].

Partly, the questionnaire consisted of the following items: satisfaction with the outcome of surgery, time span between surgery and complete recovery, and recommendation to other patients. To assess satisfaction after surgery, we asked: 'Are you satisfied with the result of the surgery?' The answer was measured on a 5-point Likert scale ranging from very satisfied to very dissatisfied. In the analysis, we dichotomized this into very satisfied/satisfied, and moderately satisfied/dissatisfied/very dissatisfied. We also asked patients if they would recommend the sacrospinous hysteropexy to other women with a prolapse. The answers could be yes, no, or do not know. To evaluate the time until complete recovery, we asked: 'How quickly did you feel completely recovered from surgery?' The answer was measured on a 5-point Likert scale ranging from within 2 weeks, 2-4 weeks, 1-3 months, 3-6 months, and more than 6 months.

Urogenital symptoms were measured with the Urogenital Distress Inventory (UDI), which was validated for the Dutch population [11]. In this validation study on a large population-based sample, it was shown that the domain construction of the Dutch version was different from the original one. The following five domains were identified: urinary incontinence, overactive bladder, pain, obstructive micturition, and prolapse. The scores of these domains vary from 0 to 100. A high score on a particular domain indicates more bothersome symptoms.

Disease-specific quality of life was measured with the Incontinence Impact Questionnaire (IIQ), also validated for the Dutch population [11]. These questions covered the following five domains: physical functioning, mobility, emotional functioning, social functioning, and embarrassment. Again, the score ranged between 0 (best quality of life) and 100 (worst quality of life).

We used information from the medical files to compare the baseline characteristics of the group of responders with the nonresponders. We also compared the characteristics of the women who underwent the gynecological examination with the group of responders who did not. By doing so, we analyzed if there were differences in baseline or outcome parameters between responders and nonresponders that could have influenced our findings.

\section{Statistical analysis}

Descriptive statistics were used for the whole population. To compare differences between groups, a Student's $t$ test was used for continuous data and the $\chi^{2}$-test for nominal or ordinal data. The significance level was set at an $\alpha$ of 0.05 . Statistical analysis was performed with the Statistical Package for the Social Sciences (SPSS) 12.0 for Windows.

\section{Results}

Of the 133 women, a total of 99 women responded to the questionnaire. Sixty of these 99 women underwent a gynecological examination. Mean follow-up was 22.8 months (3-55). In Table 1, the baseline characteristics of the responders and nonresponders to the questionnaire are shown.

In Table 2, the complications during and after surgery are shown. Buttock pain occurred in $15 \%$ of the women but resolved spontaneously in all but two women. One woman complained of severe buttock pain directly after surgery based on entrapment of the branches of the ischiadic nerve. 
Table 1 Patient characteristics of responders and nonresponders to the questionnaire

\begin{tabular}{|c|c|c|c|}
\hline Patient characteristics & Responders $(n=99)$ & Nonresponders $(n=34)$ & $p$ value \\
\hline Age (years) $)^{\mathrm{a}}$ & $59.2(13.1)$ & $68.3(14.1)$ & 0.001 \\
\hline Operation for prolapse in medical history & $4(4.4 \%)$ & $3(8.8 \%)$ & NS \\
\hline Operation for urinary incontinence in medical history & $1(1.0 \%)$ & $0(0.0 \%)$ & NS \\
\hline Urinary incontinence before surgery & $36(36.4 \%)$ & $20(58.8 \%)$ & 0.002 \\
\hline Urge incontinence & $8(8.1 \%)$ & $5(14.7 \%)$ & NS \\
\hline Stress incontinence & $17(17.2 \%)$ & $10(29.4 \%)$ & NS \\
\hline Combination stress and urge incontinence & $9(9.1 \%)$ & $3(8.8 \%)$ & NS \\
\hline Unknown type & $2(2.0 \%)$ & $2(5.9 \%)$ & NS \\
\hline Fecal incontinence before surgery & $4(4.0 \%)$ & $2(6.1 \%)$ & NS \\
\hline \multicolumn{4}{|l|}{ Gynecological examination before surgery } \\
\hline Descensus uterus stage 2 or more & $90(91 \%)$ & $32(94 \%)$ & NS \\
\hline Cystoceles stage 2 or more & $79(79.8 \%)$ & $30(88.2 \%)$ & NS \\
\hline Rectoceles stage 2 or more & $22(23.9 \%)$ & $16(50 \%)$ & 0.006 \\
\hline Enterocele & $5(5.4 \%)$ & $5(15.6 \%)$ & NS \\
\hline SSF + anterior or posterior colporrhaphy & $88(88.9 \%)$ & $32(94.1 \%)$ & NS \\
\hline Additional surgery for urinary stress incontinence & $11(11.1 \%)$ & $3(8.8 \%)$ & NS \\
\hline Follow-up time in months ${ }^{\mathrm{a}}$ & $22.5(3-55)$ & $23.6(3-54)$ & NS \\
\hline
\end{tabular}

Data are numbers $(\%)$ or mean (standard deviation).

NS, not significant $(p>0.05)$

${ }^{\mathrm{a}}$ Mean (standard deviation)

The suture from the sacrospinous ligament to the cervix was replaced and the pain resolved. The other woman also had buttock pain after surgery. In this case, it was unclear if this pain was related to the surgery or related to her neurological problems in the lumbar region. After a complete evaluation, she chose to undergo a vaginal hysterectomy. After this surgery, she still experienced buttock pain, although it was less severe. One woman complained of numbness in the introitus probably based on damage to the posterior labial nerve. In one woman, postoperative bleeding occurred that needed surgical intervention. A blood transfusion was not indicated.
Based on information from the medical files, 3 women out of the $133(2.3 \%)$ had surgical treatment because of a symptomatic recurrent prolapse (one cystocele and two descensus uteri). In one case, an abdominal sacrocolpopexy of the uterus was performed. One patient had a colpocleisis (uterus in situ), and in one woman, the recurrent prolapse was corrected by a colporrhaphy anterior. Another three women were also diagnosed with a recurrent prolapse of the uterus but did not need surgical treatment.

When we look at the results of the 60 women who underwent gynecological examination, 18 women $(30 \%)$ had a cystocele at stage 2 , and 5 women $(8 \%)$ had a cystocele

Table 2 Complications related to surgery assessed from medical files

\begin{tabular}{lccc}
\hline Patient characteristics & Responders $(n=99)$ & Nonresponders $(n=34)$ & $p$ value \\
\hline Complications during surgery & $8(8.1 \%)$ & $3(8.8 \%)$ & NS \\
Blood loss $>500 \mathrm{cc}$ & $6(6.1 \%)$ & $2(5.9 \%)$ & $\mathrm{NS}$ \\
Lesion of the rectum wall & $1(1.0 \%)$ & $0(0.0 \%)$ & $\mathrm{NS}$ \\
Complications after surgery & $42(43.4 \%)$ & $10(29.4 \%)$ & $\mathrm{NS}$ \\
Deep venous thrombosis & $0(0 \%)$ & $1(2.9 \%)$ & $\mathrm{NS}$ \\
Buttock pain & $16(16.2 \%)$ & $4(11.8 \%)$ & $\mathrm{NS}$ \\
$<2$ weeks & $11(11 \%)$ & $2(25.9 \%)$ & $\mathrm{NS}$ \\
$>2$ weeks & $4(4 \%)$ & $1(2.9 \%)$ & $\mathrm{NS}$ \\
Postspinal headache & $1(1.0 \%)$ & $1(0.0 \%)$ & $\mathrm{NS}$ \\
Postoperative bleeding & $0(0.0 \%)$ & $5(14 \%)$ & $\mathrm{NS}$ \\
Urinary tract Infection & $12(12.1 \%)$ & $5(14.7 \%)$ & $\mathrm{NS}$ \\
Retention bladder & $25(25.3 \%)$ & $4(11.8 \%)$ & $\mathrm{NS}$ \\
Bladder catheterization $<2$ weeks & $24(24.2 \%)$ & $2(5.9 \%)$ & $\mathrm{NS}$ \\
Bladder catheterization $>2$ weeks & $6(6.1 \%)$ & $\mathrm{NS}$ \\
\hline
\end{tabular}

Data are numbers $(\%)$.

NS, not significant $(p>0.05)$ 
Table 3 Urogenital distress inventory and incontinence impact questionnaire

\begin{tabular}{lccc}
\hline & Gynecological examination ${ }^{\mathrm{a}}(n=60)$ & No gynecological examination ${ }^{\mathrm{a}}(n=39)$ & $p$ value \\
\hline UDI & & & \\
Urinary incontinence & $7.5(11.4)$ & $7.8(14.4)$ & $\mathrm{NS}$ \\
Overactive bladder & $14.6(17.4)$ & $16.5(23.5)$ & $\mathrm{NS}$ \\
Pain & $10.4(15.7)$ & $9.1(16.1)$ & $\mathrm{NS}$ \\
Obstructive micturition & $16.7(22.5)$ & $10.8(20.9)$ & $\mathrm{NS}$ \\
Genital prolapse & $8.2(13.0)$ & $8.1(12.6)$ & $\mathrm{NS}$ \\
IIQ & & & $\mathrm{NS}$ \\
Physical functioning & $10.6(17.5)$ & $9.5(9.2)$ & $\mathrm{NS}$ \\
Mobility & $12.6(17.2)$ & $6.3(16.4)$ & $\mathrm{NS}$ \\
Emotional health & $8.1(15.6)$ & $4.9(10.4)$ & $\mathrm{NS}$ \\
Social functioning & $6.1(12.6)$ & $5.0(11.4)$ & $\mathrm{NS}$ \\
Embarrassment & $6.9(13.3)$ & & \\
\hline
\end{tabular}

${ }^{\mathrm{a}}$ Mean (standard deviation)

NS, not significant $(p>0.05)$; UDI: $0=$ no bother, $100=$ a lot of bother; IIQ: $0=$ good quality of life, $100=$ worst quality of life

at stage 3 . Only 2 of these 23 women (8.2\%) had symptoms related to this prolapse. All women with a cystocele after surgery had an anterior wall colporrhaphy at their initial surgery. A total of 3 out of the 60 women (5\%) had a recurrence of a descensus uteri at stage 2 , but all were asymptomatic. Therefore, in total (medical file information and gynecological examination), 8 women out of $133(6 \%)$ were diagnosed with a recurrent descensus uteri. Of the 99 women who answered the questionnaire, 83 (84\%) were (very) satisfied about the results of the surgery, $91(91 \%)$ would recommend the procedure to other women, $55(56 \%)$ reported a total recovery within 3 months, and 77 (78\%) reported to be completely recovered in 6 months.

In Table 3, the results of the UDI and IIQ scores are shown for all women who responded to the questionnaire, divided in those who had a gynecological examination and those who did not. There were no statistically significant differences in UDI/IIQ scores and baseline characteristics between these groups. On a scale between $0-100$, it is clear that both the UDI and IIQ scores are in the low range, indicating little bother.

In Table 4, we compare the results of the UDI and IIQ domains for women with $(n=23)$ or without $(n=37)$ a cystocele $\geq$ stage 2 at gynecological examination at the time of follow-up. No statistically significant differences in UDI/ IIQ scores and baseline characteristics between these groups were found.

\section{Discussion}

In this study, we set out to assess the clinical outcome, complications, patients' satisfaction, and quality of life after a sacrospinous hysteropexy. At a mean follow-up of 2 years, a recurrence of the descensus uteri was rare, the satisfaction rate high, and almost all women would recommend the procedure to others. We did find a high percentage of recurrent cystoceles, but surprisingly, this did not result in a

Table 4 Urogenital distress inventory and incontinence impact questionnaire for patients who underwent gynecological examination

\begin{tabular}{lccc}
\hline & Cystocele stage $\geq 2^{\mathrm{a}}(n=23)$ & No cystocele ${ }^{\mathrm{a}}(n=37)$ & $p$ value \\
\hline UDI & & & \\
Urinary incontinence & $7.5(9.0)$ & $7.5(12.8)$ & $\mathrm{NS}$ \\
Overactive bladder & $13.5(17.7)$ & $15.3(17.4)$ & $\mathrm{NS}$ \\
Pain & $12.1(16.0)$ & $9.3(15.6)$ & $\mathrm{NS}$ \\
Obstructive micturition & $18.1(20.7)$ & $8.8(23.9)$ & $\mathrm{NS}$ \\
Genital prolapse & $8.4(13.7)$ & $13.9(20.5)$ & $\mathrm{NS}$ \\
IIQ & & $14.1(20.2)$ & $\mathrm{NS}$ \\
Physical functioning & $5.8(10.8)$ & $9.5(18.3)$ & $\mathrm{NS}$ \\
Mobility & $10.4(11.5)$ & $7.8(14.8)$ & $\mathrm{NS}$ \\
Emotional health & $6.1(10.6)$ & $8.8(15.6)$ & $\mathrm{NS}$ \\
Social functioning & $3.5(8.0)$ & $\mathrm{NS}$ \\
Embarrassment & $4.2(8.4)$ & \\
\hline
\end{tabular}

${ }^{\mathrm{a}}$ Mean (standard deviation)

NS, not significant $(p>0.05)$; UDI: $0=$ no bother, $100=$ a lot of bother; IIQ: $0=$ good quality of life, $100=$ worst quality of life 
higher degree of bother from urogenital symptoms as compared to women without a recurrent cystocele. Especially, complaints of genital prolapse symptoms did not differ between women with or without a recurrent cystocele.

After our initial study in which we retrospectively compared the sacrospinous hysteropexy with a vaginal hysterectomy as surgical procedure for descensus uteri, we now present more long-term follow-up data on a next consecutive group of patients. The strength of our study is that we used validated questionnaires to assess urogenital symptoms and disease-specific quality of life. A potential draw back is that we did not have complete follow-up data on all women. As we have shown, women who responded to the questionnaire were significantly younger and had less urinary incontinence problems as compared to the nonresponders. Age might be a factor that made women decide not to participate in the follow-up, but we cannot rule out that they had recurrent complaints. In this case, it would have been likely that the general practitioner had referred the woman, but it is also possible that the women were referred to another hospital. Another source of bias could be that not all women who responded to the questionnaire appeared for a gynecological examination. However, we showed that the results from baseline assessment and the questionnaires did not differ between these groups; thus, we do not think these results are biased.

The recurrence rate of prolapse reported in the literature after sacrospinous ligament fixation of the vaginal vault is $18 \%$ [2]. Less information is available about prolapse after sacrospinous hysteropexy. Recurrence rates vary between 6.7 (only with regard to descensus uteri) to $26 \%$ (total recurrence of descensus uteri and cystocele/rectocele) [7-9, 12]. In the group of 60 women who underwent gynecological examination, only 3 had a recurrent descensus uteri at stage 2 . As with the majority of women with a recurrent cystocele, these women did not experience symptoms of prolapse. Data from our clinical files and gynecological examination showed that a recurrence of symptomatic uterine prolapse needing surgical treatment is rare ( 2 out of 133 women).

In the literature, several complications of sacrospinous ligament fixation are documented, especially buttock pain. In our study, buttock pain occurred in $15 \%$ of the women, which is also comparable with earlier studies [13]. In almost all cases, this buttock pain resolved spontaneously within 2 weeks and could be treated with analgesics. Two women needed repeated surgery to release the suture.

Fifty-six percent of women completely recovered within 3 months and $78 \%$ within 6 months. This is comparable to the group of women who underwent a sacrospinous hysteropexy in the study of van Brummen et al. [9]. In this study, women who underwent a sacrospinous hyster- opexy recovered significantly faster as compared to the women who underwent a vaginal hysterectomy.

When we assessed urogenital symptoms and diseasespecific quality of life, the scores were low, suggesting a high quality of life and little complaints of urogenital problems after the sacrospinous hysteropexy. These data have to be viewed with caution because we have no information on the UDI and IIQ scores before surgery. Therefore, if there has been a significant improvement in quality of life after surgery, this cannot be derived from our data. However, in contrast to our expectations, we found that women with a recurrent cystocele after a sacrospinous hysteropexy did not have more genital prolapse complaints. It is becoming more and more obvious that the prevalence of genital prolapse in the general population is high. In a recent Dutch population-based study, the prevalence of a stage 2 or higher genital prolapse was reported to be $40 \%$ [14]. Most of these women had no serious complaints and did not seek medical attention. Therefore, a 35\% prevalence of recurrent cystoceles after surgery might well be comparable to the normal 'physiological' situation in the population.

Several potential limitations of our study need to be discussed. First, there might have been a selection bias. We tried to avoid this as much as possible by emphasizing the importance of the woman's cooperation, regardless the presence of symptoms. There might have been another selection bias in the group of patients appearing for gynecological examination. It is unclear if this group had the best anatomical results or had doubts about the anatomical results and therefore wanted this check up. However, as we have indicated, no differences in baseline and questionnaire outcomes were found between these groups.

Second, there might have been an indication bias. Women who chose specifically for this operation instead of the more common vaginal hysterectomy probably had high expectations of this procedure. This might have had influenced their answers. There also could have been an indication bias of the gynecologist who selected the women for this procedure.

Third, although a mean follow-up of 2 years is reasonable, it could have been that some recurrences were not yet developed at the time of gynecological examination or research of medical files. However, all women who were treated for their recurrent prolapse were diagnosed within 4 to 6 months after initial surgery.

Fourth, our data on the recovery time after surgery were collected retrospectively and are, therefore, subject to recall bias by the patient. Therefore, these data have to be interpreted with caution.

Fifth, objective measures such as urodynamics or pad testing were not performed after surgery to confirm urinary incontinence and detrusor hyperactivity. However, these objective measures are known to correlate moderately with the reported symptoms $[15,16]$. 
Finally, a great part of our data was collected retrospectively from medical files.

We used a unilateral sacrospinous fixation, and one could argue that a bilateral fixation might improve the results. However, one study showed that bilateral sacrospinous fixation was only possible in $73 \%$ of women with vaginal vault prolapse and in $56 \%$ of women who underwent a vaginal hysterectomy at the same procedure [17]. In addition, it is unclear if bilateral fixation will give better results, and it might be associated with more complications and more postoperative buttock pain. Apart from the technical difficulty in attaching the cervix close to both ligaments, our results of the unilateral fixation show that the chance of symptomatic recurrence is very low. We therefore advocate the use of sacrospinous hysteropexy unilateral.

The number of recurrent cystoceles, although often asymptomatic, remains a point of concern in all classical prolapse surgical techniques. The use of synthetic or allograft implants might reduce the risk of recurrent cystoceles. Observational data are encouraging, but there is an urgent need for well-designed randomized trails, comparing classical reefing techniques with the use of implants.

We think that uterine preservation during prolapse surgery is a good option for women who desire this. Again, due to a lack of randomized trials, it is unclear if preserving the uterus gives better results and a higher quality of life compared the traditional vaginal hysterectomy, the latter being the standard in Dutch practice for many years. At this time, a randomized trial between these two surgery methods is performed.

In conclusion, sacrospinous hysteropexy is a safe procedure for the primary treatment of descensus uteri. Recurrence of a uterine prolapse is rare. The vast majority of women are highly satisfied with the outcome and would recommend this surgery to others. The majority of women with recurrent cystoceles do not have complaints about it, so reintervention should not be based on anatomical grounds solely.

Acknowledgments The author would like to thank J. den Boon, Isala Clinics Zwolle, The Netherlands and C.I.M. Aalders, Rijnstate Hospital Arnhem, The Netherlands.

\section{References}

1. Carey MP, Slack MC (1994) Transvaginal sacrospinous colpopexy for vault and marked uterovaginal prolapse. Br J Obstet Gynaecol 101:536-540
2. Sze EH, Karram MM (1997) Transvaginal repair of vault prolapse: a review. Obstet Gynecol 89:466-475

3. Nieminen K, Huhtala H, Heinonen PH (2003) Anatomical and functional assessment and risk factors of recurrent prolapse after vaginal sacrospinous fixation. Acta Obstet Gynecol Scand $82: 471-478$

4. Kovac SR, Cruikshank SH (1993) Successful pregnancies and vaginal deliveries after sacrospinous uterosacral fixation in five of nineteen patients. Am J Obstet Gynecol 168:1778-1786

5. Rane A, Lim YN, Withey G, Muller R (2004) Magnetic resonance imaging findings following three different vaginal vault prolapse repair procedures: a randomised study. Aust NZ J Obstet Gynaecol 44:135-139

6. Sze EH, Meranus J, Kohli N, Miklos JR, Karram MM (2001) Vaginal configuration on MRI after abdominal sacrocolpopexy and sacrospinous ligament suspension. Int Urogynecol J Pelvic Floor Dysfunct 12:375-379

7. Hefni MA, El-Toukhy TA (2006) Long-term outcome of vaginal sacrospinous colpopexy for marked uterovaginal and vault prolapse. Eur J Obstet Gynecol Reprod Biol 127:257-263

8. Hefni MA, El Toukhy TA, Bhaumik J, Katsimanis E (2003) Sacrospinous cervicocolpopexy with uterine conservation for prolapse in elderly women: An evolving concept. Am J Obstet Gynecol 188:645-650

9. Van Brummen HJ, van de Pol G, Aalders CIM, Heintz APM, van der Vaart CH (2003) Sacrospinous hysteropexy compared to vaginal hysterectomy as primary surgical treatment for a descensus uteri: effects on urinary symptoms. Int Urogynecol J 14:350-355

10. Bump RC, Mattiasson A, Bo K, Brubaker LP, DeLancey JO, Klarskov P (1996) The standardization of terminology of female pelvic organ prolapse and pelvic floor dysfunction. Am J Obstet Gynecol 175:10-17

11. Van der Vaart CH, de Leeuw JRJ, Roovers JPWR, Heintz APM (2003) Measuring Health-related quality of life in women with urogenital dysfunction: the urogenital distress inventory and incontinence impact questionnaire revisited. Neurourol Urodyn 22:97-104

12. Maher CF, Cary MP, Slack MC, Murray CJ, Milligan M, Schluter P (2001) Uterine preservation or hysterectomy at sacrospinous colpopexy for uterovaginal prolapse? Int Urogynecol J 12:381-384

13. Karram MM, Walters MD (1993) Pelvic organ prolapse: enterocele and vaginal vault prolapse. Mosby, St. Louis, pp 245-252

14. MCP Slieker-ten Hove, Vierhout M, Bloembergen H, Schoemaker G (2004) Distribution of POP in the general population; prevalence, severity, etiology and relation with the function of the pelvic floor muscles. ICS, Paris

15. Ryhammer AM, Laurberg S, Djurhuus JC, Hermann AP (1998) No relationship between subjective assesment of urinary incontinence and pad test weight gain in a random population sample of menopausal women. J Urol 159:800-803

16. Wyman JF, Choi SC, Harkins SW, Wilson MS, Fantl JA (2002) The urinary diary in evaluation of incontinent women: a test-retest analysis. Obstet Gynecol 71:812-817

17. Pohl JF, Frattarelli JL (1997) Bilateral transvaginal sacrospinous colpopexy: preliminary experience. Am J Obstet Gynecol 177 (6):1356-1361 